\title{
The X-ray Light-Curves and CME onset of a M2.5 flare of July 6, 2006
}

\author{
J. E. Mendoza-Torres ${ }^{1}$ and J. E. Pérez-León ${ }^{2}$ \\ ${ }^{1}$ Instituto Nacional de Astrofísica Optica y Electrónica, \\ C. Luis Enrique Erro No 1, Tonantzintla, Pue., México \\ email: mend@inaoep.mx \\ ${ }^{2}$ UANL, Monterrey, Nuevo León, México \\ email: enrique584@hotmail.com
}

\begin{abstract}
A M2.5 solar flare observed by RHESSI in the 6-100 keV range on July 6, 2006 led to a Coronal Mass Ejection (CME). Two compact sources at 12-100 keV are seen at the beginning of the flare, whose further evolution fits well in a loop. Also, time-profiles of the flare at radio wavelengths are compared. The X-ray light-curves at different bands in the $6-100 \mathrm{keV}$ range and radio time profiles show some peaks superimposed on smooth variations. The aim of this work is to compare the X-ray light-curves, of fluxes integrated over the whole source, with the physical parameters of the sources of the flare. Yashiro and Gopalswamy (2009) have found that the fraction of flares that produce CME increases with the flare energy. Here, we look for the characteristics of an M2.5 flare that could make it a generator of a CME. The idea is, in future works, to look in the light-curves of similar flares at other stars for these features. It is found that the CME onset takes place around the time when an X-ray source at $12-25 \mathrm{keV}$ of Chromospheric evaporation stagnates at the loop apex, before the main peak at the light-curve at $25-50 \mathrm{keV}$ and at the radio emission curves. Probably, the amount of evaporated plasma could play some role in triggering the CME.
\end{abstract}

Keywords. Sun: X-rays, Sun: flares, Sun: coronal mass ejections (CMEs)

\section{Introduction}

It is widely accepted that flares are caused by magnetic reconnection somewhere in the Corona, where particles are accelerated and part of them precipitate to lower layers. Radio emission is expected to arise due to gyrosynchrotron emission near the loop feet, while X-ray emission arises when the downstream reaches the Chromosphere. For this reason, it is frequently observed that the time variations of the flux at microwaves are similar to X-ray light-curves, in particular the peaks, although with time delays (Liu et al. 2015). However, the electron populations that generate peaks and smooth variations could be different from each other (Lin \& Johns 1993). The precipitating electrons lead to Chromospheric Evaporation, filling the magnetic loop with heated plasma. Then, the $\mathrm{X}$-ray light-curves exhibit peaks and smooth time-variations.

It has been observed (Yashiro and Gopalswamy 2009) that the fraction of flares accompanied by CMEs increases with flare energy and practically all the X-class events produce CMEs. Then, it is expected that the larger the flare energy, the higher possibilities that a CME will take place. This result could have important implications in the estimation of the mass loss of the Sun in early phases and also in stars (Drake et al. 2016). However, it is not known what characteristics of strong flares are responsible of this relation, characteristics that some weak flares may noy display.

In the last years, it has been possible to image the sources of solar X-ray flares with a 4 -second cadence. With this good time resolution, it is possible to identify details of 

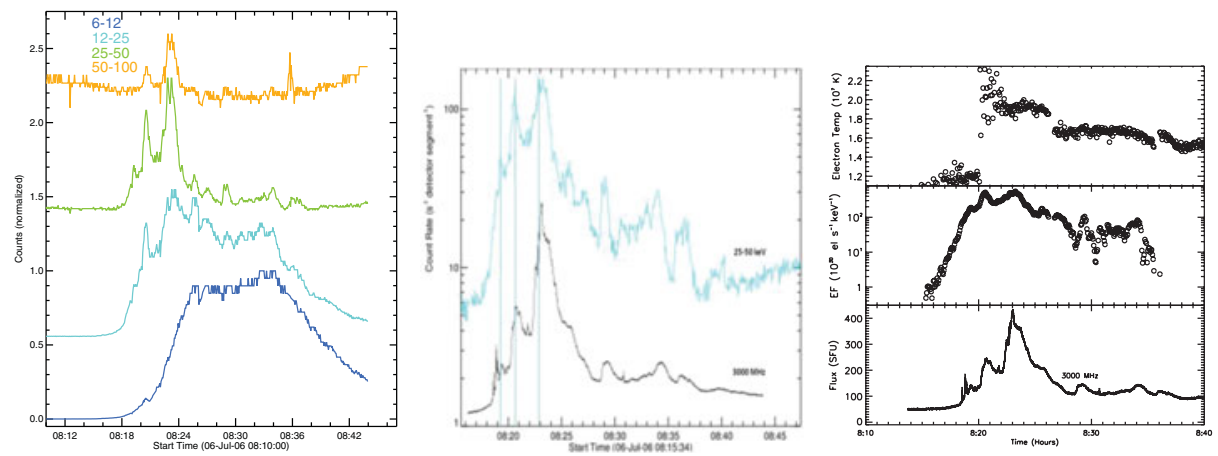

Figure 1. Left. Light-curves of the X-ray emission during the flare, from top to bottom: 50-100 $\mathrm{keV}, 25-50 \mathrm{keV}, 12-25 \mathrm{keV}$ and 6-12 keV. Middle. The time profiles observed at Ondrejov at $3 \mathrm{GHz}$ and at RHESSI at 25-50 keV. Right. Electron Temperature (upper panel) and Electron Flux (middle panel) obtained from the spectra of the integral field. Time profile observed at Ondrejov at $3 \mathrm{GHz}$ (lower panel).
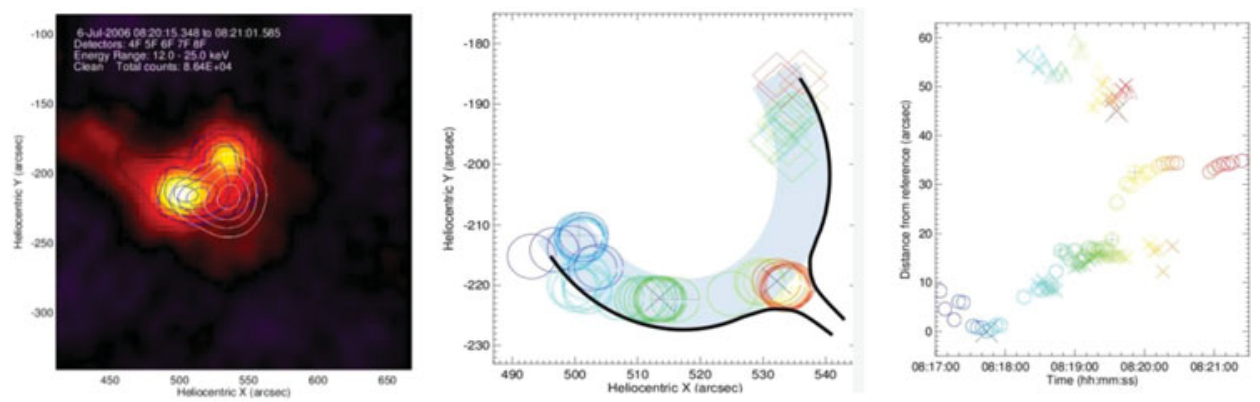

Figure 2. Left. X-ray sources observed by RHESSI at $12-25 \mathrm{keV}$, the image corresponds to 08:20 UT, black contours to 08:21 UT, blue to 08:22: UT and white to 08:29 UT. Middle. The $12-25 \mathrm{keV}$ locations of the sources at different times of the flare, circles are for the South source and triangles for the North one. Right. Distance from the Southern loop foot to the $12-25 \mathrm{keV}$ sources, as seen at different times.
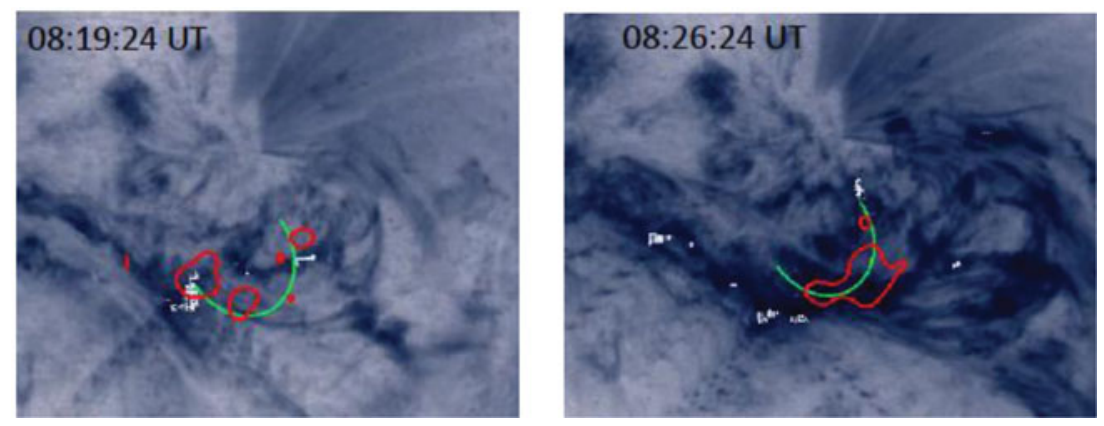

Figure 3. TRACE $171 \mathrm{~A}$ images with the times given in each panel. The green arc denotes the loop fitted to the $12-25 \mathrm{keV}$ sources and the red contours the sources. Left. The sources at different locations of the loop. Right. The source at the loop apex when the CME is triggered.

the time evolution of the flare sources and their spectra and light-curves. We estimate physical parameters, at different times of the flare, from the X-ray spectra of the emission integrated over the whole field and for the spectra of three different Regions of Interest (ROIs) which are located at the loop feet and at the loop apex. 
The X-ray light-curves, integrated over the RHESSI field, are compared with those obtained for the ROIs. Also, the time profiles of the Electron Temperature and Emission Measure (EM) obtained from the integrated spectra are compared with the corresponding time-curves obtained for the ROIs.

\section{Data and Results}

The M2.5 flare began at about 08:18 UT, as observed by RHESSI at X-ray and at radio wavelengths at San Vito and Ondrejov. In the right-panel of Figure 1, the light-curves at different energy bands in the $6-100 \mathrm{keV}$ range are shown. In the middle panel the 25-50 keV light-curve and $3 \mathrm{GHz}$ time profile are shown. In the right panel, the electron temperature (ET) and the electron flux (EF), for the whole image, are shown and the flux at some radio wavelengths. In Figure 2 (left) the 12-25 keV image at 18:20 UT is shown. In the middle-panel the locations of the source on the loop at different times are shown, purple corresponds to the initial times and red to the last ones. At the right-panel, the distances to the Southern source are plotted. At $\sim$ 08:20-08:22 UT the source stagnates at the loop top. The evaporating source also stagnates at a mid location between the feet and the apex, at this time a peak is seen in the light-curve.

According to the values obtained from the ROIs at the loop feet, the ET grows from $\sim 10 \mathrm{MK}$ at 08:18 UT to $\sim 50 \mathrm{MK}$ at 08:19 UT and decreases to $\sim 20 \mathrm{MK}$ at 08:20 UT, which is the time when the evaporating source stagnates at the loop apex. The Emission Measure (EM) grows during the two more intense peaks. For the ROI at the apex of the loop, the ET varies similarly to that at the feet but the EM almost linearly grows from 08:18 UT to 08:25 UT. In the integrated EM, TE and Electron Flux (EF) the time-curves show both, peaks and smooth variations (right panel of Figure 1).

Lets recall that at the apex the EM continuously grows. Then, a possible characteristic of the flares that could play a role in triggering an CME, could be the amount of plasma reaching the loop apex due to Chromospheric evaporation. We have to point out that, the estimation of the source location seems to indicate that, the source underwent various back and forth small displacements while being at the apex. However, the displacements are short compared with the size source. Nevertheless, we cannot rule out other possible causes, such as oscillations (Stepanov et al. 2010). However, in this flare, they should have to be of small amplitude.

\section{Conclusions}

The more intense peaks at the $\mathrm{X}$-ray and radio time-curves coincide with the times when the loop sources are most intense and when an evaporating source stagnates. The more intense peak occurs when the source stagnates at the loop apex. The onset of the CME takes place after this.

\section{References}

Brown, J. C., MacKinnon, A. L., Zodi, A. M., \& Kaufmann, P. 1983, A\& A, 123, 10

Drake, J. J., Cohen, O., Garraffo, C. O., \& Kashya, V. 2016, Solar and Stellar Flares and Their Effects on Planets, Proceedings IAU Symposium No. 320

Lin, R. P. \& Johns, C. M. 1993, ApJ, 417, 53L

Liu, Z-Y., Li, Y-P., Gan, W-Q., \& Firoz K. A. 2015, Research in Astronomy and Astropnysics, 15,64

Stepanov A. V., Yuri T. Tsap, Y. T., \& Kopylova, Y. G. 2010, Solar and Stellar Variability: Impact on Earth and Planets, Proceedings IAU Symposium No. 264, 288

Yashiro, S. \& Gopalswamy, N. 2009, IAU Symposium, Vol. 25\%, IAU Symposium, ed. N. Gopalswamy \& D. F. Webb, 233 